\title{
Differential stem proteomics and metabolomics profiles for four wheat cultivars in response to the insect pest Wheat Stem Sawfly
}

\section{Supporting Information}

Florent D. Lavergne ${ }^{a}$, Corey D. Broeckling ${ }^{b}$, Kitty J. Brown ${ }^{b}$, Darren M. Cockrell ${ }^{c}$, Scott D. Haley $^{d}$, Frank B. Peairs ${ }^{c}$, Stephen Pearce ${ }^{d}$, Lisa M. Wolfe ${ }^{b}$, Courtney E. Jahn ${ }^{c,}$, Adam L. Heuberger ${ }^{a, d^{*}}$

a. Department of Horticulture and Landscape Architecture, Colorado State University, Fort Collins, CO 80523, USA

b. Proteomics and Metabolomics Facility, Colorado State University, Fort Collins, CO 80523, USA

c. Department of Bioagricultural Sciences and Pest Management, Colorado State University, Fort Collins, CO 80523, USA

d. Department of Soil and Crop Sciences, Colorado State University, Fort Collins, CO 80523, USA

Supporting Information File 1: Figure S1 -- Insect container setup for the WSS infestation experiment; Tables S1-S3 -- Differentially regulated proteins between control and infested plants in Conan, Denali, and Reeder. Table S5 -- Differentially regulated metabolites between control and WSS infested wheat.

Supporting Information File 2: Table S4 -- Excel document of all detected wheat stem metabolites, proteins, and their annotations.

Supporting Information File 3: Evidence for metabolite annotations as output of the interpretMSspectrum algorithm in the MSFinder program. 


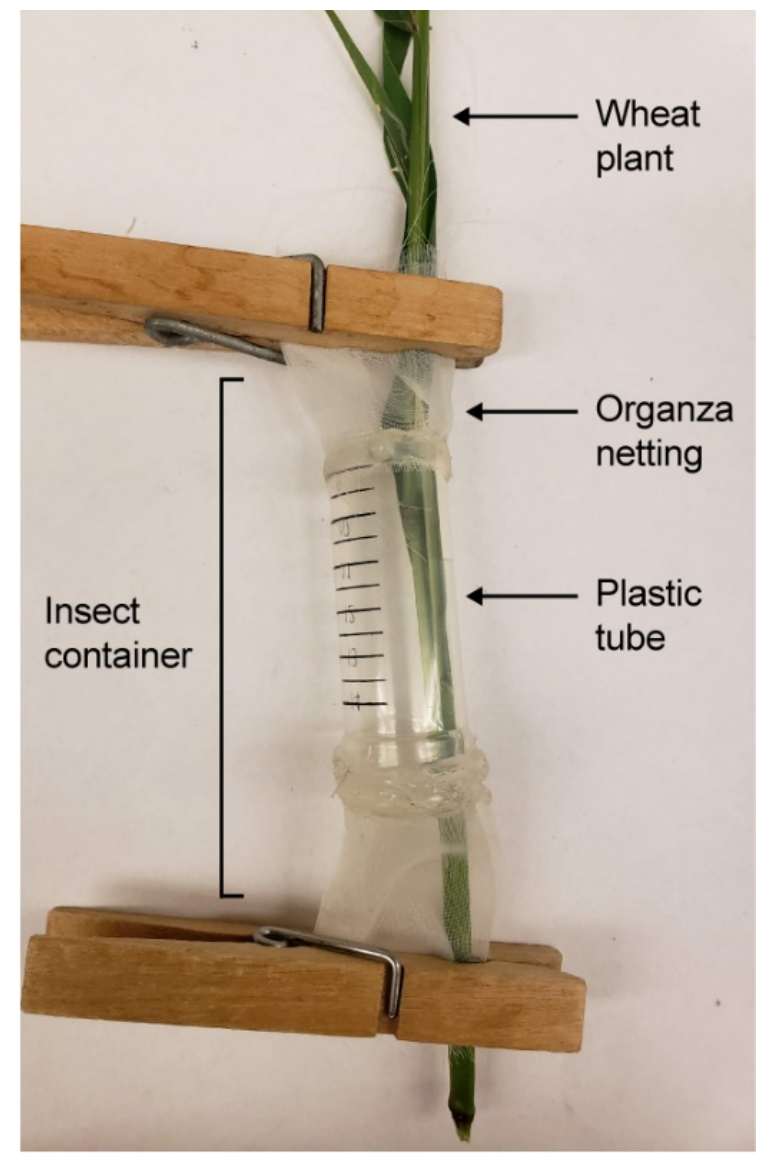

Figure S1. Insect container setup for the WSS infestation experiment. A $5 \mathrm{~cm}$ long $\times 1.5 \mathrm{~cm}$ diameter plastic tube with organza netting on both openings was placed around wheat stems to contain female WSS and control for oviposition location. Insects were left for egg deposition within the container area for ten days. 
Table S1. Differentially regulated proteins between control and infested plants in Conan. Table description is as described for Table 1.

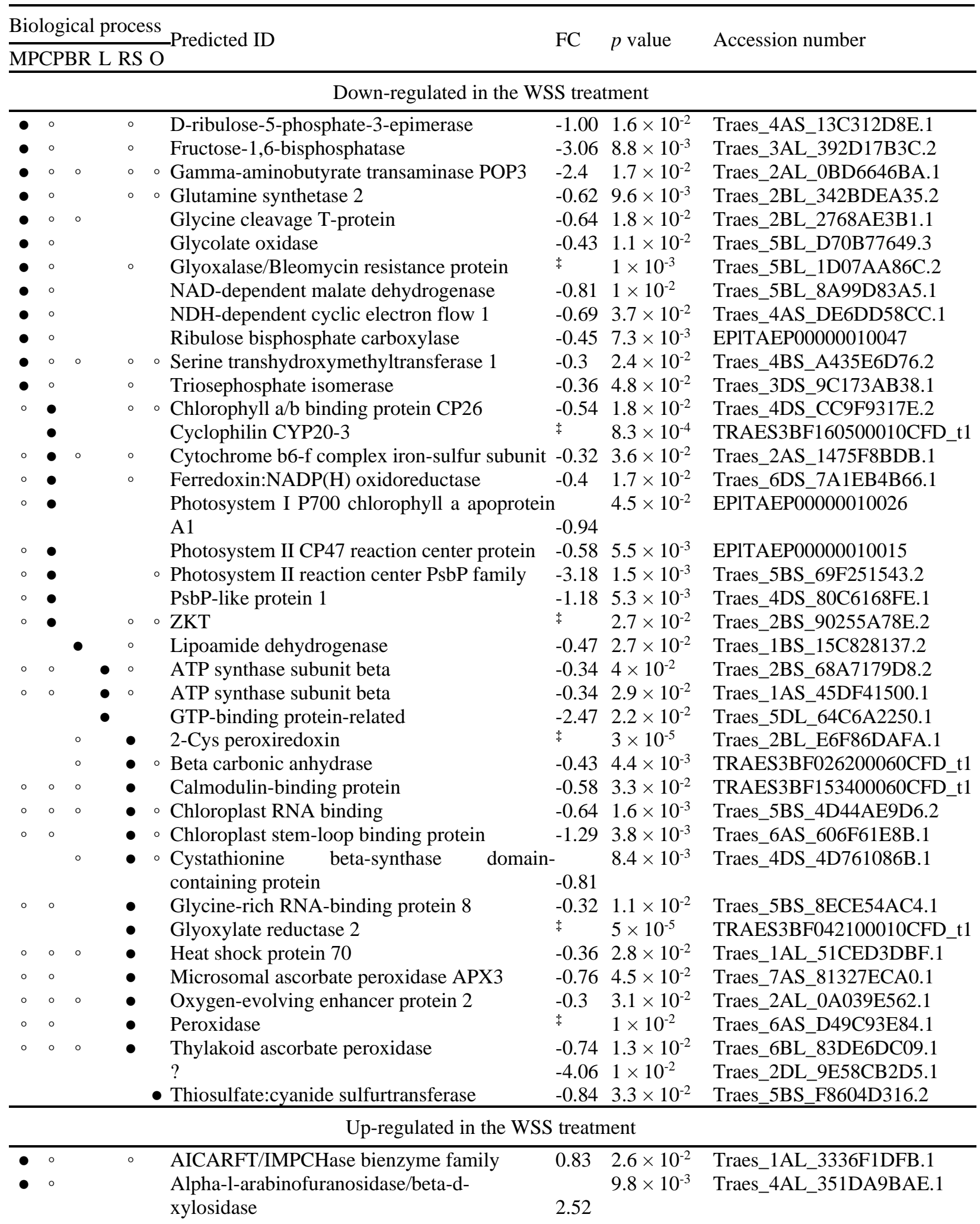




\begin{tabular}{|c|c|c|c|c|c|c|c|}
\hline & 。 & & & Biotin synthetase & $\neq$ & $6.8 \times 10^{-3}$ & Traes_3AL_45EF63187.1 \\
\hline & 。 & & & GDP-D-mannose 3',5'-epimerase & $\ddagger$ & $3.5 \times 10^{-3}$ & Traes_1BL_1CCF44ECE.1 \\
\hline & & & & Glutamate decarboxylase 5 & 0.86 & $6.7 \times 10^{-3}$ & TRAES3BF050800360CFD_t1 \\
\hline & 。 & & 。 & - Glycyl-tRNA synthetase & $\ddagger$ & $1.5 \times 10^{-2}$ & Traes 7DS 817122161.1 \\
\hline & 。 & & 。 & - NADP-ME2 & $\neq$ & $2 \times 10^{-5}$ & Traes_1AS_A53D11A37.1 \\
\hline & $\circ$ & & $\circ$ & - Phosphoenolpyruvate carboxylase & 0.91 & $3.8 \times 10^{-2}$ & Traes_5BL_6642E2A8B.1 \\
\hline & 。 & & 。 & Sucrose synthase activity & 3.4 & $3.5 \times 10^{-2}$ & Traes_4AL_2BC235062.1 \\
\hline & $\circ$ & & $\circ$ & Ubiquitin-activating enzyme E1 2 & 2.88 & $4.1 \times 10^{-2}$ & Traes_5DL_0BE2B1D42.2 \\
\hline$\bullet$ & $\circ$ & & & Ubiquitin-like modifier (SUMO) polypeptide & 2.75 & $5.1 \times 10^{-3}$ & TRAES3BF-091100230CFD_t1 \\
\hline & - & & & 20S proteasome beta subunit & 0.59 & $4.5 \times 10^{-2}$ & Traes_4DS_8EF070D72.1 \\
\hline 。 & - & & $\circ$ & oteasome beta subunit & 0.57 & $1.2 \times 10^{-2}$ & 058D6EF22.1 \\
\hline 。 & - & & 。 & tRNA synthetase & 1.82 & $3.1 \times 10^{-2}$ & Traes_4AL_4CF27F6B1.1 \\
\hline 。 & - & & & Cytochrome b559 subunit alpha & 0.54 & $4 \times 10^{-2}$ & EPITAEP00000010041 \\
\hline 。 & • & & & NADH-ubiquinone oxidoreductase-related & $\ddagger$ & $3.4 \times 10^{-3}$ & E44.1 \\
\hline 。 & - & & $\circ$ & Photosystem II type I chlorophyll a/b-binding & & $3.8 \times 10^{-2}$ & Traes_1AL_0F39673AF1.3 \\
\hline & & & & prot & 2.24 & & \\
\hline 。 & - & & & - Ribosomal protein L1p/L10e family & 1.19 & $7.2 \times 10^{-3}$ & Traes_4BS_4DB5737A6.1 \\
\hline ○ & - & & & nal protein L7Ae/L30e/S12e/Gadd45 & & $2.3 \times 10^{-4}$ & Traes_5DL_1299E23A4.1 \\
\hline ○ & - & & $\circ$ & mal protein large subunit 27 & 1.56 & $3.6 \times 10^{-2}$ & 0CFD_t1 \\
\hline 。 & & 。 & & Ribosomal protein S4 & 2.19 & $2.5 \times 10^{-2}$ & Traes_5AS_9F68 \\
\hline & $\bullet$ & & & $\begin{array}{l}\text { Sec14p-like phosphatidylinositol transfer } \\
\text { family }\end{array}$ & & $1.9 \times 10^{-2}$ & S3BF043700170CFD_t1 \\
\hline & & & & TCP-1/cpn60 chaperonin family & 2.58 & $3.2 \times 10^{-2}$ & Traes_7AL_F3ED3303D.1 \\
\hline & - & & $\circ$ & onin family protein & 1.33 & $3.7 \times 10^{-3}$ & Traes_6DL_30AD166CD.1 \\
\hline & & & & & & $8.2 \times 1$ & A.1 \\
\hline & $\circ$ & & & eavy chain 1 & $\neq$ & $3.8 \times 10^{-3}$ & Traes_4DS_EB7A5C35C.2 \\
\hline & & & & & & $2.9 \times 10^{-3}$ & C65.1 \\
\hline & & & $\circ$ & GD & ¥ & $5 \times 10^{-3}$ & \\
\hline$\circ$ & $\circ$ & $\bullet$ & & P synthase subunit B1 & 0.29 & $3.4 \times 10^{-2}$ & 39A.1 \\
\hline 。 & $\circ$ & & - & $26 \mathrm{Sp}$ & 1.18 & $3.2 \times 10^{-2}$ & Traes_2AS_DFDA79E58.1 \\
\hline 。 & $\circ$ & 。 & - & Ankyrin repeat domain-containing protein 2B & 0.69 & $3 \times 10^{-2}$ & Traes_7AS_860ACFB35.1 \\
\hline & & & & & 0.55 & $3.8 \times 10^{-2}$ & Traes_2DS_C6B631387.1 \\
\hline 。 & $\circ$ & & - & Gener: & 0.69 & $4.1 \times 10^{-2}$ & Traes_4BS_342A62CE6.1 \\
\hline 。 & 。 & & & General regulatory factor & 0.37 & $1.9 \times 10^{-2}$ & Traes_4AL_6 \\
\hline 。 & $\circ$ & & $\bullet$ & Phi glutathione transferase & 0.99 & $5.7 \times 10^{-3}$ & Traes_4BL_689C6389A.1 \\
\hline & $\circ$ & & & & 0.26 & $1.8 \times 10^{-2}$ & Traes_1AS_C0D4CD39A.2 \\
\hline & 。 & & $\circ$ & - Actin & 0.14 & $1.5 \times 10^{-2}$ & Traes_1AS_A8AD3BE99.2 \\
\hline & 。 & & 。 & ntaining protein & 1.83 & $4.7 \times 10^{-2}$ & Traes_2DL_28DFAC79D.2 \\
\hline ○ & 。 & & & & & & Traes_7BS_1F4C5C328.1 \\
\hline & & & & Aluminum induced protein & 0.55 & $4 \times 10^{-2}$ & Traes_5BL_17F1F28B6.1 \\
\hline
\end{tabular}


Table S2. Differentially regulated proteins between control and infested plants in Denali. Table description is as described for Table 1.

\begin{tabular}{|c|c|c|c|c|c|c|c|c|}
\hline \multicolumn{5}{|c|}{ Biological } & _Predicted ID & FC & $p$ value & Accession number \\
\hline \multicolumn{9}{|c|}{$\overline{\text { MPCPBR L RS O }}$} \\
\hline \multicolumn{9}{|c|}{ Down-regulated in the WSS treatment } \\
\hline - & $\circ$ & & & 。 & - Cinnamoyl CoA reductase & -0.62 & $3.5 \times 10^{-2}$ & Traes_5DL_D93D4EE55.1 \\
\hline • & $\circ$ & & & & • Geranylgeranyl reductase & & $3.4 \times 10^{-2}$ & Traes_6DL_0FF72D765.1 \\
\hline$\bullet$ & $\circ$ & & & & - Glutamate-1-semialdehyde 2,1-aminomutase & -0.69 & $1.9 \times 10^{-2}$ & Traes_7AS_9C283FE66.1 \\
\hline$\bullet$ & $\circ$ & $\circ$ & & $\circ$ & Glycolate oxidase & -0.47 & $4.4 \times 10^{-2}$ & Traes_2AL_C51BB91CC.3 \\
\hline • & $\circ$ & & & $\circ$ & • NADPH:protochlorophyllide oxidoreductase B & -1.43 & $4.3 \times 10^{-2}$ & Traes_1AL_BB3D071F8.1 \\
\hline$\circ$ & & & & $\circ$ & ○ 26S proteasome subunit RPN8a & -0.79 & $9.2 \times 10^{-3}$ & Traes_2AL_AA6937D6B.1 \\
\hline$\circ$ & $\bullet$ & & & & Cytochrome b559 subunit alpha & -1.32 & $4.4 \times 10^{-2}$ & EPITAEP00000010041 \\
\hline$\circ$ & $\bullet$ & & & & Ribosomal protein L3 family & -1.32 & $4 \times 10^{-3}$ & Traes_6AS_478618F75.1 \\
\hline$\circ$ & $\bullet$ & & & & Ribosomal protein L4 & -0.81 & $2.6 \times 10^{-2}$ & Traes_4DL_31D6228F5.1 \\
\hline \multirow[t]{3}{*}{$\circ$} & $\bullet$ & $\circ$ & $\circ$ & $\circ$ & - Thylakoid formation 1 & -0.81 & $1.7 \times 10^{-2}$ & Traes_2DS_D106620BE.1 \\
\hline & $\circ$ & & 。 & $\bullet$ & - Chaperone protein htpG family & -0.97 & $4.2 \times 10^{-2}$ & Traes_5AL_1DA3B4631.1 \\
\hline & $\circ$ & $\circ$ & & & 。 Chloroplast chaperonin 60 & -0.62 & $4.9 \times 10^{-2}$ & Traes_7AS_3247D97E8.2 \\
\hline 。 & $\circ$ & & & - & - Chloroplast RNA binding protein & -1.94 & $1.4 \times 10^{-2}$ & Traes_2DS_01D518748.1 \\
\hline$\circ$ & & $\circ$ & & & - Chloroplastic drought-induced stress protein & -2.06 & $4.3 \times 10^{-2}$ & Traes_6DL_C6E63ED1C.1 \\
\hline \multirow[t]{2}{*}{$\circ$} & $\circ$ & & & & Glyoxylate aminotransferase & -0.62 & $4.8 \times 10^{-2}$ & Traes_2AL_85FCE950B.1 \\
\hline & & $\circ$ & & - & - Rubisco activase & -0.71 & $1.3 \times 10^{-2}$ & Traes_4DS_5C2095EE7.2 \\
\hline
\end{tabular}

\begin{tabular}{|c|c|c|c|c|c|c|c|}
\hline \multicolumn{8}{|c|}{ Up-regulated in the WSS treatment } \\
\hline - & $\circ$ & ○ & 。 & - 26S proteasome AAA-ATPase subunit RPT4a & 1.25 & $3.2 \times 10^{-2}$ & Traes 7AL EA1DE71E6.1 \\
\hline$\bullet$ & $\circ$ & & & 3-keto-acyl-CoA thiolase 2 precursor & 0.85 & $1.9 \times 10^{-2}$ & Traes_6DL_7960654CF.2 \\
\hline • & $\circ$ & & $\circ$ & - Aconitase & 0.37 & $1.5 \times 10^{-2}$ & Traes_6AL_28CCA7F5D.1 \\
\hline & $\circ$ & & 。 & - Aconitase & 0.32 & $3.9 \times 10^{-2}$ & Traes_6BL_4704FF2B4.1 \\
\hline & $\circ$ & & $\circ$ & Aconitase 1 & 0.86 & $1.5 \times 10^{-2}$ & Traes_4DL_3D374FF4C.1 \\
\hline • & $\circ$ & & & Aldolase & 0.21 & $1.8 \times 10^{-2}$ & Traes_3AL_441C0AE1B.1 \\
\hline$\bullet$ & $\circ$ & $\circ$ & $\circ$ & Aldolase-type TIM barrel family protein & 0.38 & $4.6 \times 10^{-2}$ & TRAESSBBF-142600080CFD_t1 \\
\hline 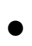 & $\circ$ & & & Aspartate aminotransferase 3 & 1.02 & $1.3 \times 10^{-3}$ & Traes_3DL_870617108.1 \\
\hline • & $\circ$ & $\circ$ & & Betaine aldehyde dehydrogenase & 0.55 & $4.9 \times 10^{-2}$ & Traes_6AL_42AEB5299.1 \\
\hline 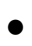 & $\circ$ & & & - Dehydroquinate-shikimate dehydrogenase & 2.9 & $6.1 \times 10^{-3}$ & Traes_5AS_908BF5D38.1 \\
\hline & $\circ$ & $\circ$ & $\circ$ & Enolase & 0.39 & $2 \times 10^{-2}$ & Traes_5AS_116663495.1 \\
\hline & $\circ$ & $\circ$ & 。 & - Glycosyltransferase & $\ddagger$ & $2.5 \times 10^{-3}$ & Traes_7BL_0E93FBCFF.2 \\
\hline & $\circ$ & $\circ$ & ○ & $\begin{array}{l}\text { Hydroxycinnamoyl-Coenzyme } \\
\text { shikimate/quinate hydroxycinnamoyltransferase }\end{array}$ & $\begin{array}{l}\mathrm{A} \\
\mathrm{e} 2.23\end{array}$ & $2.5 \times 10^{-2}$ & Traes_6AL_D8A91F983.1 \\
\hline & $\circ$ & & $\circ$ & Lactate/malate dehydrogenase & $\ddagger$ & $4 \times 10^{-5}$ & Traes_6DS_5DBF48560.1 \\
\hline & $\circ$ & & $\circ$ & Mitochrondrion targeted citrate synthase & 0.75 & $4.1 \times 10^{-2}$ & Traes_6BS_682497935.1 \\
\hline & & & & NAD(P)-linked oxidoreductase & 0.52 & $1.1 \times 10^{-2}$ & Traes_2BL_2FD539228.2 \\
\hline & $\circ$ & $\circ$ & $\circ$ & NADP+-isocitrate dehydrogenase & 0.69 & $4.4 \times 10^{-2}$ & Traes_3AL_3D5C860FD.1 \\
\hline & $\circ$ & & & N-carbamoylputrescine amidase & $\ddagger$ & $4.8 \times 10^{-4}$ & Traes_5DS_01D241425.1 \\
\hline & $\circ$ & & & Nitrilase/cyanide hydratase & 2.49 & $4.7 \times 10^{-2}$ & Traes_4AS_CDEB9D532.2 \\
\hline & $\circ$ & & $\circ$ & $\begin{array}{l}\text { Orotate phosphoribosyltransferase/orotidine-5' } \\
\text { phosphate decarboxylase }\end{array}$ & 2.71 & $2.2 \times 10^{-2}$ & TRAES3BF139100030CFD_t1 \\
\hline & $\circ$ & & $\circ$ & - Phosphoenolpyruvate carboxylase & 0.77 & $4.6 \times 10^{-2}$ & Traes_5BL_6642E2A8B.1 \\
\hline & $\circ$ & & & Pyrophosphorylase 2 & 0.54 & $3.3 \times 10^{-2}$ & Traes_6AL_7E70268F1.2 \\
\hline & $\circ$ & $\circ$ & 。 & Spermine synthase & $\ddagger$ & $1.7 \times 10^{-2}$ & Traes_1DL_43FED2619.1 \\
\hline & $\circ$ & $\circ$ & & Ubiquitin-like modifier (SUMO) polypeptide & 2.32 & $3.7 \times 10^{-2}$ & TRAES3BF-̄91100230CFD_t1 \\
\hline 。 & $\bullet$ & & & 20S proteasome beta subunit PBB2 & 0.36 & $2.5 \times 10^{-2}$ & Traes_1BS_548536A26.2 \\
\hline 。 & $\bullet$ & $\circ$ & ○ & - 26S proteasome AAA-ATPase subunit RPT3 & 3.18 & $3.2 \times 10^{-2}$ & Traes_2AL_80D15B4FB.1 \\
\hline$\circ$ & • & & $\circ$ & 60S acidic ribosomal protein P2 & $\ddagger$ & $4.7 \times 10^{-4}$ & Traes_6AS_D49C93E84.1 \\
\hline
\end{tabular}




\begin{tabular}{|c|c|c|c|c|c|c|c|}
\hline & - & & $\circ$ & Cysteine proteinase & $\begin{array}{l}0.85 \\
\ddagger\end{array}$ & $\begin{array}{l}1.7 \times 10^{-2} \\
8 \times 10^{-5}\end{array}$ & Traes_5DL_AC7C885A0.1 \\
\hline & & & & & & & \\
\hline & $\circ$ & 。 & $\circ$ & GDP dissociation inhibitor & 3.16 & $2.1 \times 10^{-2}$ & Traes_1DL_401D433BE.1 \\
\hline \multirow[t]{2}{*}{ 。 } & 。 & 。 & & - Pleckstrin homology (PH) domain superfamily & 1.12 & $2.8 \times 10^{-2}$ & Traes_1AL_06BC2ED91.1 \\
\hline & & & & Rab1 GTPase & 0.51 & $4.5 \times 10^{-2}$ & Traes_3DL_4B8AB34A9.1 \\
\hline \multirow[t]{2}{*}{$\circ$} & $\circ$ & & ○ & $\begin{array}{l}\text { - Vacuolar ATP synthase subunit } \\
\text { Annexin }\end{array}$ & $\begin{array}{l}0.49 \\
0.62\end{array}$ & $\begin{array}{l}1.3 \times 10^{-2} \\
4.7 \times 10^{-2}\end{array}$ & $\begin{array}{l}\text { Traes_7DL_D4B6FF473.1 } \\
\text { Traes 7AS FFB7CAFC3.1 }\end{array}$ \\
\hline & $\circ$ & & & - Chiti & $\neq$ & $9.8 \times 10^{-4}$ & Traes_6DS_AA77F7548.1 \\
\hline$\circ$ & $\circ$ & & & $\begin{array}{l}\text { protein } 90 \\
\text { lrial aldehyde dehydrogenase }\end{array}$ & $\begin{array}{l}0.3 \\
0.54\end{array}$ & $\begin{array}{l}2.5 \times 10^{-2} \\
1.3 \times 10^{-2}\end{array}$ & $\begin{array}{l}\text { Traes_7DS_CB359539B.1 } \\
\text { Traes_7AS_DE1248005.1 }\end{array}$ \\
\hline$\circ$ & $\circ$ & & • & Peroxidase & $\neq$ & $1.3 \times 10^{-2}$ & Traes_2DL_1CB540BCC.3 \\
\hline c & $\circ$ & & & Phi glutathione transferase & 0.45 & $7.5 \times 10^{-3}$ & Traes_1AL_1B8FDA3D4.1 \\
\hline 。 & 。 & 。 & - & • Phytochelatin synthase & 2.08 & $4.6 \times 10^{-2}$ & Traes_4DL_A6F0CFF85.1 \\
\hline & $\circ$ & 。 & P & - Protein disulfide-isomerase & 0.79 & $2.9 \times 10^{-2}$ & Traes_4DS_26272902A.1 \\
\hline$\circ$ & $\circ$ & $\circ$ & - & - Sulfite reductase & 0.94 & $2 \times 10^{-2}$ & Traes_1AL_5A7E85C4E.1 \\
\hline & $\circ$ & $\circ$ & $\circ$ & - DC1 c & 0.96 & $3.8 \times 10^{-2}$ & Traes_2DL_28DFAC79D.2 \\
\hline & $\circ$ & $\circ$ & 0 & - Receptor fo & 0.8 & & 30CFD_t1 \\
\hline
\end{tabular}


Table S3. Differentially regulated proteins between control and infested plants in Reeder. Table description is as described for Table 1.

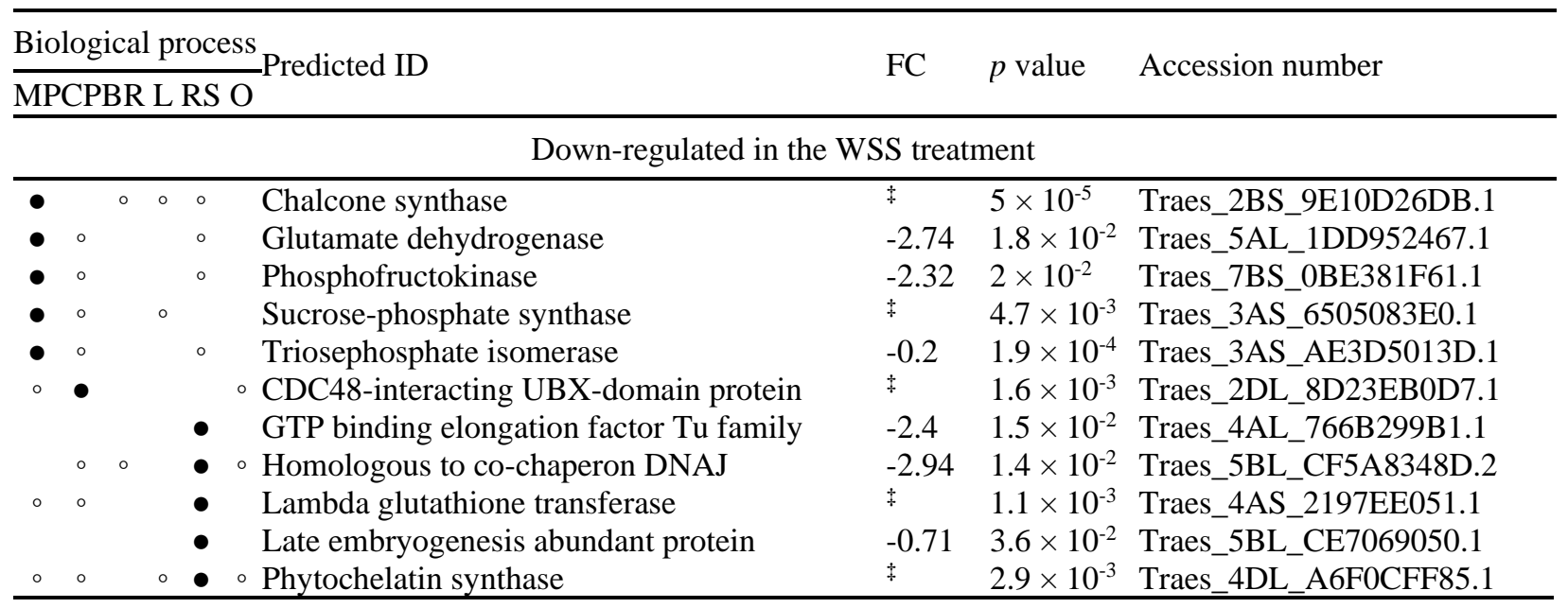

Up-regulated in the WSS treatment

\begin{tabular}{|c|c|c|c|c|c|}
\hline & \% & $\circ$ ○ Alpha-1,4-glucan-protein synthase family & 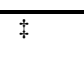 & $2 \times 10^{-2}$ & Traes_2AL_41B176D01.2 \\
\hline • & 。 & - $\quad$ Aspartate aminotransferase 5 & 0.56 & $4.4 \times 10^{-2}$ & Traes_6DL_961B6AFC5.1 \\
\hline & 。 & Dehydroascorbate reductase 1 & $\ddagger$ & $4 \times 10^{-5}$ & Traes_7AS_0BB13DD79.1 \\
\hline$\bullet$ & $\circ$ & D-ribulose-5-phosphate-3-epimerase & 0.85 & $2.6 \times 10^{-2}$ & Traes_4AS_13C312D8E.1 \\
\hline - & $\circ$ & 。 $\quad$ Fructose-bisphosphate aldolase 1 & 0.34 & $9.3 \times 10^{-3}$ & Traes_4BS_D12DBE6D3.1 \\
\hline • & 。 & - $\quad$ Fructose-bisphosphate aldolase 2 & 0.43 & $1.2 \times 10^{-2}$ & Traes_5DS_D693664F7.4 \\
\hline$\bullet$ & & $\begin{array}{l}\text { Glyceraldehyde 3-phosphate dehydrogenase } \\
\text { subunit } 2\end{array}$ & A 0.2 & $4 \times 10^{-2}$ & Traes_2BL_5D64E8C87.1 \\
\hline - & 。 & Glyceraldehyde-3-phosphate dehydrogenase & 0.6 & $2.1 \times 10^{-2}$ & Traes_4DL_F394FF94A.1 \\
\hline • & 。 & Pinoresinol res & $\ddagger$ & $2.2 \times 10^{-2}$ & Traes_2BS_23E0AC565.2 \\
\hline • & $\circ$ & - Triosephosphate isomerase & 0.54 & $2.9 \times 10^{-2}$ & Traes_5BL_D5F2A5070.1 \\
\hline & $\bullet$ & 50S ribosomal protein L12-C & 1.1 & $4.9 \times 10^{-2}$ & TRAES3BF028000060CFD_t1 \\
\hline 。 & - & Cysteine proteinase & 0.62 & $3 \times 10^{-2}$ & Traes_5DL_AC7C885A0.1 \\
\hline 。 & - & omplex of photosystem II & 0.43 & $1.8 \times 10^{-2}$ & Traes_2BS_4AE914BE2.1 \\
\hline 。 & - & Ribul & 0.46 & $3.5 \times 10^{-2}$ & Traes_5AL_1 \\
\hline 。 & • & Rubis & 0.39 & $4.6 \times 10^{-2}$ & Traes $2 \mathrm{AS}$ \\
\hline 。 & - & Thyls & 1.3 & $4.8 \times 10^{-2}$ & Traes_2AS_D \\
\hline 。 & $\bullet$ & ribonucleoprotein A & $\ddagger$ & $1.1 \times 10^{-2}$ & Traes_4AL_8C7636C36.1 \\
\hline & & $\circ \quad \circ$ RNA-binding $\mathrm{f}$ & 2.56 & $1.5 \times 10^{-2}$ & Traes_5DL_63AB65800.2 \\
\hline & & buso cubunit D & 0.66 & $4.3 \times 10^{-2}$ & Traes_2AL_2B01693491.2 \\
\hline & & Vacuolar membrane ATPAse complex & 1.6 & $3.3 \times 10^{-2}$ & Traes_2AL_152FE9E5F.1 \\
\hline 。 & 。 & - Beta-d-xylosidase & 0.72 & $2 \times 10^{-2}$ & Traes_2AL_3E4F9. \\
\hline & & tancoreconcivo family & & $3.8 \times 10^{-2}$ & Traes_4DS_2347 \\
\hline & & - $\quad$ Ear & & $2.8 \times 10^{-2}$ & Traes 2D \\
\hline 。 & 。 & - $\circ$ Fumarate hydratase 1 & & $8.9 \times 10^{-3}$ & Traes_4AS_2DCA42965.1 \\
\hline & & - $\circ \mathrm{Met}$ & 0.7 & $2.5 \times 10^{-2}$ & Traes_4DS_AFAF2A29E.1 \\
\hline 。 & 。 & kinase type 1 & 0.42 & $2.3 \times 10^{-2}$ & Traes_1AL_6F2E87864.2 \\
\hline 。 & 。 & - $\quad$ Phi glutathione transferase & 1.5 & $3.3 \times 10^{-2}$ & Traes 3DL B1E3D279A.1 \\
\hline
\end{tabular}


Table S5. Differentially regulated metabolites between control and WSS infested wheat. ${ }^{a}$

\begin{tabular}{|c|c|c|c|c|c|c|}
\hline Superclass $^{b}$ & Subclass $^{c}$ & Chemical name (abbr...) $)^{d}$ & $\mathrm{RT}^{e}$ & $m / z^{f}$ & $p$ value $^{g}$ & \\
\hline \multicolumn{7}{|c|}{ Down-regulated in the WSS treatment - Hatcher } \\
\hline Alkaloid/der. & Harmala alkaloid & 1-(9H- $\beta$-Carbolin-1... & 204 & 422.1744 & $7.7 \times 10^{-3}$ & $\bullet^{\circ}$ \\
\hline \multirow{6}{*}{ Lipid/lipid-like cmp. } & Taxane/der. & (2aR,4S,4aS,5R,6R,8S,9aS... & 309 & 650.294 & $2.7 \times 10^{-2}$ & \\
\hline & Saccharolipid & 2-[4- $\left(\left\{\left[\left(2 \mathrm{R}^{*}, 3 \mathrm{E}, 4 \mathrm{R}^{*}\right)-3\right.\right.\right.$-ethyl... & 225 & 910.3106 & $4.2 \times 10^{-2}$ & \\
\hline & Diterpenoid & 4 -[Formyl(methyl)amino]... & 340 & 468.3094 & $2.7 \times 10^{-2}$ & \\
\hline & $\begin{array}{l}\text { 1-acyl-sn-glycero-3- } \\
\text { phosphocholine }\end{array}$ & LPC(18:3) & 452 & 517.3178 & $2.4 \times 10^{-2}$ & \\
\hline & Trihydroxy acid & - & 331 & 586.2915 & $3 \times 10^{-2}$ & \\
\hline & Hydroxysteroid & - & 313 & 806.3362 & $1.3 \times 10^{-2}$ & • \\
\hline \multirow{4}{*}{ Organic acid/der. } & Tetracarboxylic acid & $(1 \mathrm{R}, 3 \mathrm{~S}, 4 \mathrm{~S}, 5 \mathrm{~S}, 6 \mathrm{R}, 7 \mathrm{R})-4,7 \ldots$ & 267 & 666.2885 & $4 \times 10^{-2}$ & \\
\hline & Tetracarboxylic acid & Decipinin A & 290 & 600.2207 & $3.4 \times 10^{-2}$ & \\
\hline & Oligopeptide & - & 260 & 372.2118 & $3.2 \times 10^{-2}$ & \\
\hline & Keto acid/der. & - & 354 & 248.1073 & $4.8 \times 10^{-2}$ & \\
\hline \multirow{3}{*}{ Organic oxygen cmp } & $\begin{array}{l}\text { Aminocyclitol } \\
\text { glycoside }\end{array}$ & $(1 \mathrm{R}, 2 \mathrm{~S}, 3 \mathrm{~S}, 4 \mathrm{~S}, 5 \mathrm{~S}, 6 \mathrm{R})-2,3,4 \ldots$ & 28 & 355.1485 & $5.1 \times 10^{-3}$ & $\bullet$ \\
\hline & Glucuronic acid der. & 2,3,5-trihydroxy-4-methoxy... & 335 & 224.0531 & $3.5 \times 10^{-2}$ & \\
\hline & $\begin{array}{l}\text { Monosaccharide } \\
\text { phosphate }\end{array}$ & 2-Deoxy-6-O-phosphono... & 46 & 246.0511 & $3.5 \times 10^{-2}$ & \\
\hline \multirow{3}{*}{$\begin{array}{l}\text { Organoheterocyclic } \\
\text { cmp. }\end{array}$} & Indolizidine & - & 300 & 434.1245 & $1.3 \times 10^{-3}$ & - \\
\hline & 6-aminopurine & 9-( $\beta$-D-Erythro-pentofuran... & 300 & 265.081 & $5.9 \times 10^{-3}$ & - \\
\hline & Isoindolone & (2R,2'R,4'aS,6'R,8'aS)-7-Aceton... & 264 & 441.2505 & $2.1 \times 10^{-2}$ & $\bullet$ \\
\hline \multirow{3}{*}{$\begin{array}{l}\text { Phenylpropanoid/ } \\
\text { polyketide }\end{array}$} & Cinnamic acid ester & \{2-[4-(Hydroxymethyl)-2... & 288 & 396.1574 & $4.8 \times 10^{-2}$ & \\
\hline & Coumarin/der. & Scoparon & 379 & 206.0588 & $4.6 \times 10^{-2}$ & \\
\hline & $\begin{array}{l}\text { Flavonoid-7-O- } \\
\text { glycoside }\end{array}$ & Veno-V & 222 & 608.1732 & $3.9 \times 10^{-2}$ & \\
\hline \multirow{5}{*}{ - } & $\begin{array}{l}\text { Benzoxazinoid } \\
\text { glycoside }\end{array}$ & DIBOA-glucoside & 106 & 343.0913 & $7.5 \times 10^{-3}$ & $\bullet$ \\
\hline & Benzodioxole & Fagaramide & 61 & 247.1213 & $4 \times 10^{-2}$ & \\
\hline & - & 4-\{[(3R,4R)-4-(4-Hydroxy-3... & 225 & 520.1936 & $7.9 \times 10^{-3}$ & - \\
\hline & - & (1S,3aS,4R,6aR)-1,4-Bis... & 246 & 536.1899 & $1.4 \times 10^{-2}$ & \\
\hline & - & Propanamide, N... & 248 & 555.2224 & $4.3 \times 10^{-2}$ & \\
\hline \multicolumn{7}{|c|}{ Up-regulated in the WSS treatment - Hatcher } \\
\hline \multirow{4}{*}{ Benzenoid } & Tyrosol/der. & Propanoic acid 4-hydroxy... & 240 & 194.0946 & $1.4 \times 10^{-2}$ & $\bullet$ \\
\hline & Benzenesulfonyl cmp. & 4-(4-isopropoxyphenyl)... & 221 & 292.0779 & $3 \times 10^{-2}$ & \\
\hline & Benzene & 4,4-dimethyl-2-phenyl... & 32 & 175.1005 & $3.1 \times 10^{-2}$ & \\
\hline & Methoxyphenol & - & 383 & 208.0743 & $8.6 \times 10^{-3}$ & - \\
\hline \multirow{3}{*}{ Lignan } & Neolignan & Methyl (2E)-3-(4-\{[(1R,2R)-1,3... & 285 & 404.1488 & $1.6 \times 10^{-3}$ & $\bullet$ \\
\hline & Furanoid lignan & $\left(1 \mathrm{~S}, 2 \mathrm{~S}, 1^{\prime} \mathrm{S}, 2^{\prime} \mathrm{S}\right)-2,2^{\prime}-\{(1 \mathrm{R}, 3 \mathrm{aS} \ldots$ & 359 & 810.3093 & $3.1 \times 10^{-2}$ & \\
\hline & & Huazhongilexin & 286 & & $6.2 \times 10^{-3}$ & \\
\hline \multirow{6}{*}{ Lipid/lipid-like cmp. } & Fatty alcohol ester & [(2Z,8Z)-10-hydroxydeca-2,8... & 337 & 204.0789 & $2.1 \times 10^{-2}$ & $\bullet$ \\
\hline & Methionine/der. & (2S)-2-amino-4-dimethyl... & 224 & 163.0673 & $4 \times 10^{-2}$ & \\
\hline & Triterpenoid & Microtropioside B & 370 & 502.3147 & $4 \times 10^{-2}$ & \\
\hline & Guaianolide/der. & - & 277 & 376.1521 & $1.9 \times 10^{-2}$ & - \\
\hline & $\begin{array}{l}\text { Colensane/clerodane } \\
\text { diterpenoid }\end{array}$ & - & 322 & 806.3351 & $3.8 \times 10^{-2}$ & \\
\hline & Xanthophyll & - & 417 & 738.3807 & $4.8 \times 10^{-2}$ & \\
\hline \multirow{3}{*}{ Nucleoside } & $\begin{array}{l}\text { 1-ribosyl-imidazole } \\
\text { carboxamide }\end{array}$ & - & 23 & 380.073 & $9.9 \times 10^{-5}$ & $\bullet$ \\
\hline & Pyrimidine & & & & & \\
\hline & $\begin{array}{l}\text { ribonucleoside } \\
\text { monophosphate }\end{array}$ & - & 23 & 542.1261 & $1.2 \times 10^{-2}$ & $\bullet$ \\
\hline
\end{tabular}




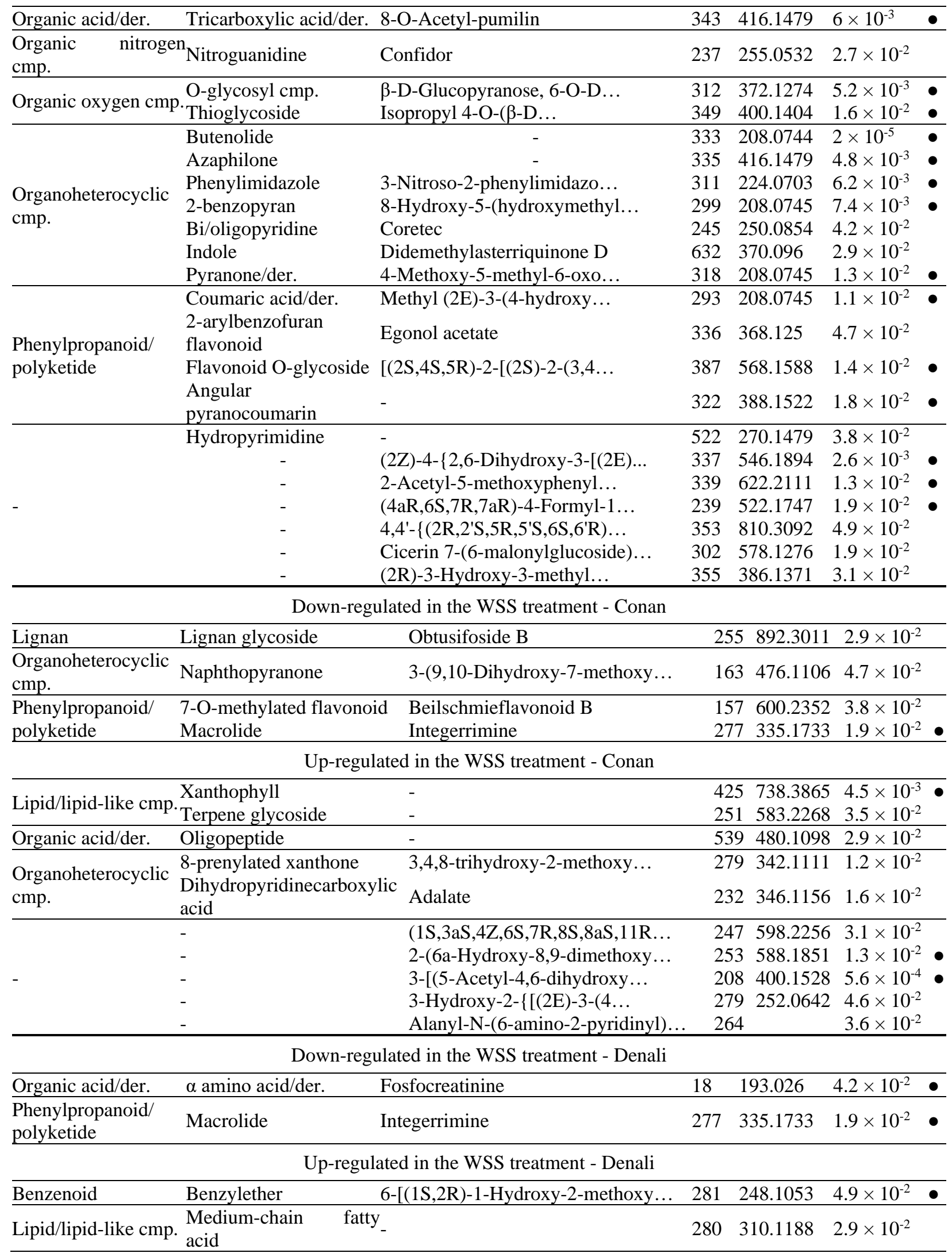




\begin{tabular}{|c|c|c|c|c|c|c|}
\hline Nucleoside & $\begin{array}{l}\text { Pyrimidine } \\
\text { ribonucleoside } \\
\text { monophosphate }\end{array}$ & - & 23 & 542.1261 & $2.7 \times 10^{-3}$ & $\bullet$ \\
\hline $\begin{array}{l}\text { Organoheterocyclic } \\
\text { cmp. }\end{array}$ & $\begin{array}{l}\text { Carbazole } \\
\text { Dioxolopyran }\end{array}$ & $\begin{array}{l}\text { (2'S,3'S,4R)-2,2"-Diimino-2'... } \\
\text { (2,2,7,7-Tetramethyltetrahydro... }\end{array}$ & $\begin{array}{r}418 \\
209 \\
\end{array}$ & $\begin{array}{l}508.2338 \\
353.1146 \\
\end{array}$ & $\begin{array}{l}1.8 \times 10^{-2} \\
1.1 \times 10^{-2} \\
\end{array}$ & $\bullet$ \\
\hline $\begin{array}{l}\text { Phenylpropanoid/ } \\
\text { polyketide }\end{array}$ & $\begin{array}{l}\text { Flavonoid-7-O- } \\
\text { glycoside } \\
\text { 7-O-methylated } \\
\text { flavonoid }\end{array}$ & $\begin{array}{l}{[(2 \mathrm{R}, 3 \mathrm{~S}, 4 \mathrm{R}, 5 \mathrm{R}, 6 \mathrm{~S})-6-[(2 \mathrm{~S}, 3 \mathrm{R}, 4 \mathrm{~S} \ldots} \\
\text { Beilschmieflavonoid B }\end{array}$ & 236 & 708.1907 & $\begin{array}{l}10^{-4} \\
1.2 \times 10^{-3}\end{array}$ & $\bullet$ \\
\hline & $\begin{array}{l}- \\
- \\
-\end{array}$ & $\begin{array}{l}\text { 3-[(5-Acetyl-4,6-dihydroxy... } \\
\text { Isopropyl 2-methoxyethyl... }\end{array}$ & $\begin{array}{r}208 \\
269 \\
\end{array}$ & $\begin{array}{l}400.1528 \\
418.1733 \\
\end{array}$ & $\begin{array}{l}5.6 \times 10^{-4} \\
2.4 \times 10^{-3} \\
\end{array}$ & $\bullet$ \\
\hline \multicolumn{7}{|c|}{ Down-regulated in the WSS treatment - Reeder } \\
\hline Organic acid/der. & $\begin{array}{l}\text { Benzenesulfonamide } \\
\alpha \text { amino acid/der. } \\
\alpha \text { amino acid amide } \\
\end{array}$ & $\begin{array}{l}\text { 4-(3-amino-1H-indazol-5-yl)-N... } \\
\text { (3R,6S)-3-Hydroxy-3-(3-hydroxy... } \\
\text { Tert-butyl (2S)-2-carbamoyl... }\end{array}$ & $\begin{array}{l}206 \\
266 \\
66\end{array}$ & $\begin{array}{l}344.1302 \\
424.1388 \\
228.1476 \\
\end{array}$ & $\begin{array}{l}2.6 \times 10^{-2} \\
2.1 \times 10^{-2} \\
2.7 \times 10^{-2} \\
\end{array}$ & $\bullet$ \\
\hline $\begin{array}{l}\text { Organoheterocyclic } \\
\text { cmp. }\end{array}$ & $\begin{array}{l}\text { Porphyrin } \\
\text { Phtalide } \\
\text { Hydroxypyrimidine }\end{array}$ & $\begin{array}{l}\text { - } \\
\text { Salvianduline C } \\
\text { Thymine 5-methyluracil }\end{array}$ & $\begin{array}{l}280 \\
224 \\
63\end{array}$ & $\begin{array}{l}648.2717 \\
400.1531 \\
126.0434 \\
\end{array}$ & $\begin{array}{l}2.4 \times 10^{-2} \\
3.7 \times 10^{-2} \\
4 \times 10^{-2} \\
\end{array}$ & $\bullet$ \\
\hline $\begin{array}{l}\text { Phenylpropanoid/ } \\
\text { polyketide }\end{array}$ & Coumaric acid/der. & Methyl \{(1R,2R)-2-[(2Z)-5... & 256 & 248.1045 & $\frac{2.3 \times 10^{-2}}{4.8 \times 10^{-3}}$ & $\bullet$ \\
\hline \multicolumn{7}{|c|}{ Up-regulated in the WSS treatment - Reeder } \\
\hline Organic oxygen cm & $\begin{array}{l}\text { Monosaccharide } \\
\text { phosphate }\end{array}$ & - & 259 & 344.1343 & $4.1 \times 10^{-2}$ & \\
\hline & - & $\begin{array}{l}\text { (1aR,3aS,5aR,6S,9S,10aS,10bR)... } \\
\text { Athrombin K }\end{array}$ & $\begin{array}{l}150 \\
242\end{array}$ & $\begin{array}{l}494.2884 \\
308.1043\end{array}$ & $\begin{array}{l}4.1 \times 10^{-2} \\
4.4 \times 10^{-2}\end{array}$ & \\
\hline
\end{tabular}

${ }^{a}$ Compounds were classified based on their regulation in the WSS treatment and superclass. Abbr... = abbreviated name, full names are available in Table S1; RT = retention time; $\mathrm{m} / \mathrm{z}=$ massto-charge ratio; VIP = variable importance for the projection; der. = derivative; cmp. = compound. ${ }^{b}$ Superclasses correspond to the second highest level of ontology based on the ClassyFire algorithm.

'Subclasses correspond to the lowest level of ontology based on the ClassyFire algorithm.

${ }^{d}$ Compounds were assigned chemical names using the International Union of Pure and Applied Chemistry (IUPAC) nomenclature.

${ }^{e}$ Compound retention times were measured based on the time of elution from the chromatography column.

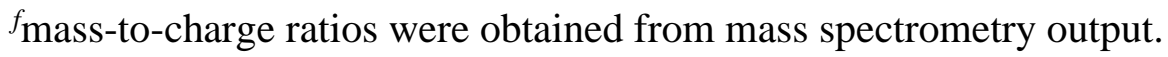

${ }^{g} p$ values were calculated from $t$ tests (factors of treatment) and adjusted by a Benjamini-Hochberg correction.

${ }^{h}$ Metabolite with high variable importance of the projection (VIP) score in the OPLS model. 